\title{
CARACTERÍSTICAS PSICOSOCIALES DE ADOLESCENTES CON INTENTO DE SUICIDIO
}

\author{
Kelly Saucedo ${ }^{1}$ \\ Karina Martínez \\ Papsivi- Minsalud \\ Gloria Reyes
}

\section{Resumen}

La presente investigación tuvo como objetivo describir las características psicosociales de adolescentes con intento de suicidio durante el año 2017, víctimas y no víctimas del conflicto armado. Se indagaron los casos registrados por la Secretaria de Salud Departamental —bajo los principios éticos y de confidencialidad-, encontrándose 49 adolescentes con intento de suicidio en el municipio de Sincelejo, 43 mujeres y 6 hombres, cuyas edades oscilaban entre los 12 y 17 años, de los cuales, 20 fueron víctimas del conflicto armado. La investigación fue de tipo descriptivo, con un diseño no experimental, de corte transversal. El análisis de los datos se realizó de manera descriptiva empleando el programa SPSS. En los resultados obtenidos se evidenció que el mayor número de intentos de suicidio se encuentra en el sexo femenino dentro del rango de 12 a 15 años de edad. El tipo de intento con mayor prevalencia fue la intoxicación con $61,25 \%$. Asimismo, prevalecieron los intentos previos de suicidio en $34,7 \%$, el trastorno depresivo con $22,4 \%$ y los problemas de pareja en el 10,2\% de la población estudiada.

1 Especialista en procesos familiares y comunitarios. Psicóloga. Correo: Kellysau_89@ hotmail.com.

2 Especialista en procesos familiares y comunitarios. Psicóloga. Correo Correo: Luzkary1983@gmail.com

3 Especialista en procesos familiares y comunitarios. Psicóloga. Correo: Paoreyes18@ hotmail.com. 
Estas son las principales características psicosociales asociadas a la problemática del suicidio en adolescentes.

Palabras clave: intento de suicidio, adolescencia, victimas, conflicto armado.

\section{Abstract}

The purpose of this paper was to describe the psychosocial characteristics of adolescents with suicide attempts during 2017, victims and non-victims of the armed conflict. The cases registered by the Departmental Health secretary were investigated under the ethical and confidential principles, finding 49 adolescents attempting suicide in the municipality of Sincelejo, 43 women and 6 men, whose ages ranged were from 12 to 17 years of age, from which 20 were victims of the armed conflict. The research is descriptive, with a nonexperimental, cross-sectional design. Data analysis was performed descriptively using the SPSS program. In the results obtained it was evidenced that, the greatest number of suicide attempts is in the female sex within the range of 12 to 15 years of age. The type of attempt with the highest prevalence was intoxication (with 61.25\%). Likewise, previous suicide attempts prevailed in $34.7 \%$, depressive disorder with $22.4 \%$ and couple problems in $10.2 \%$ of the population studied. These are the main psychosocial characteristics associated with the problem of suicide in adolescents.

Keywords: attempted suicide, adolescence, victims, armed conflict. 


\section{Introducción}

En Colombia según datos estadísticos del Instituto Nacional de Salud, durante el año 2017 se registraron 9.214 intentos de suicidio en el país, la mayoría de los casos se presentaron en adolescentes entre los 15 y los 19 años (29,1\%) (El Mundo.com, 2017). En el Departamento de Sucre, en la ciudad de Sincelejo, de acuerdo a datos estadísticos de la Secretaria de Salud Departamental ${ }^{4}$, para el mismo año se registraron 332 intentos de suicidio y 49 casos, específicamente en adolescentes entre los 12 y 17 años de edad. Este panorama resulta aún más preocupante, si se tiene en cuenta que 20 adolescentes fueron identificados como víctimas del conflicto armado, situación que visibiliza una problemática latente en esta población.

Ante tal situación, resulta necesario hacer énfasis en esta etapa de la vida y estudiar las características psicosociales que podría presentar este grupo de adolescentes vulnerables al intento de suicidio. Del mismo modo, es importante analizar si existe alguna relación al comparar este fenómeno en adolescentes víctimas y no víctimas del conflicto armado, teniendo en cuenta que la violencia es uno de los eventos que afecta directamente la salud mental de los seres humanos sin distinción alguna. Las ideas precedentes impulsaron la presente investigación.

Inicialmente se revisó el artículo denominado "Suicidio y trastorno mental" realizado en Colombia por Bedoya y Montaño, (2016), quienes indican en sus hallazgos que la presencia de un trastorno mental constituye un factor de riesgo para cometer un suicidio; entre los trastornos más asociados como riesgo del suicidio se encuentran la depresión y la esquizofrenia. La investigación señala que es imperativo abordar la problemática con prioridad, dado que se considera un problema de salud pública que se ha incrementado en la sociedad colombiana con múltiples implicaciones en la esfera personal, familiar y social. Por otro lado, sugiere que cuando se presenten intentos de suicidio, se debe realizar un estricto seguimiento y acompañamiento de profesionales idóneos con un plan de acción que vincule a la familia en el proceso de recuperación del paciente.

4 Información obtenida por las investigadoras en la Secretaria de Salud respectiva. 
Este fenómeno también fue objeto de estudio en Cuba, donde la mortalidad por suicidio ocupa el noveno lugar en la isla, por ello, Corona, Hernández y García (2016) en su estudio muestran que en los países de medianos y bajos ingresos existe mayor porcentaje de muertes por suicidio a nivel mundial, muy a pesar de que en la actualidad hay más conocimiento sobre el tema; de igual manera, este comportamiento suicida se asocia a unos factores de riesgo que se clasifican en individuales, comunitarios, familiares e institucionales. En la medida que se identifiquen de manera oportuna se pueden intervenir, fortaleciendo factores protectores, lo cual incidiría positivamente en la prevención de esta problemática y en una posible reducción de muertes por suicidio.

La problemática objeto de la presente investigación se fundamenta en el modelo ecológico de las relaciones humanas de Bronfenbrenner. Esta visión concibe al ambiente ecológico como una serie de estructuras representadas en diferentes niveles, en donde cada uno contiene al otro y tiene una función específica, el autor les da el nombre de: microsistema, mesosistema, exosistema y macrosistema. Este modelo explica el desarrollo del individuo a través de la interacción con los distintos contextos en los cuales se desenvuelve el sujeto desde una perspectiva integradora donde se intercambia información y se relaciona con el ambiente para así constituir características que permiten estructurar en cierta medida la personalidad; además, este modelo plantea que el desarrollo de los niños, niñas y adolescentes es un proceso lleno de complejidad en el que influyen múltiples factores asociados a los ambientes: micro, meso, exo y macro (Bronfenbrenner, citado por el Observatorio del Bienestar de la Niñez, OBN, 2017).

Es preocupante el aumento de las cifras de suicidio en el departamento de Sucre, Restrepo, Romero-Acosta, Verhelst-Montenegro (2019), pues los autores hallaron un incremento entre los años 2015 y 2016. Todos los esfuerzos que se realicen para sentar la línea base de la situación del suicidio en el departamento o para desarrollar estrategias de intervención resultan necesarios a fin de disminuir las cifras de suicidios en esa localidad, sobre todo en la población juvenil. Considerando estos planteamientos, el presente estudio aborda este fenómeno con el objetivo general de determinar las características psicosociales de adolescentes de 12 a 17 años de edad, víctimas y no víctimas del conflicto armado. Para lograr dicho cometido fue 
necesario describir las características del intento de suicidio e identificar aspectos psicológicos de los adolescentes seleccionados intencionalmente en el estudio.

\section{Metodología}

Diseño y tipo de investigación: El diseño de la investigación es no experimental, ya que no existe manipulación deliberada de las variables y no se tiene influencia ni control sobre ellas (Hernández, Fernandez y Baptista 2010). Por el tipo de fuente de la cual se obtuvieron los datos, responde a un diseño documental, pues se recurrió al registro de documentos para obtener información sobre el suicidio en adolescentes en la cuidad de Sincelejo. La investigación es de carácter descriptivo y transversal.

Procedimiento: El grupo de investigación está conformado por tres profesionales psicólogas que seleccionaron la temática objeto de investigación, teniendo en cuenta el alto índice de intentos de suicidio en adolescentes de la ciudad de Sincelejo.

Se acudió a la Secretaria de Salud y se solicitó autorización para utilizar los registros de dichos casos, los cuales se encuentran almacenados en una base de datos del área de salud pública de esta entidad departamental, teniendo claros los principios éticos, así como la confidencialidad dentro del trabajo investigativo y explicando de forma clara el objetivo general del estudio, pues el tema del suicidio es un problema de salud pública que se debe analizar desde el panorama internacional, nacional y local, para profundizar en sus causas y consecuencias.

Posterior a ello, se formularon los objetivos específicos, los cuales direccionan la finalidad de la investigación, que para el caso se plantearon tres; seguidamente se argumentó la relevancia social del estudio, indicando cada uno de los aportes prácticos para orientar la intervención oportuna y prevenir futuros casos. La investigación se delimitó a la ciudad de Sincelejo , considerando 49 casos presentados en adolescentes de 12 a 17 años de edad, con intento de suicidio durante el año 2017, incluyó víctimas y no víctimas del conflicto armado, tomando en cuenta que en el departamento de Sucre, se instalaron grupos al margen de la ley responsables de múltiples hechos victimizantes, lo que originó desplazamientos forzosos hacia la 
ciudad de Sincelejo, convirtiéndola en receptora de víctimas del conflicto armado.

El presente estudio consideró 18 investigaciones que sirvieron como antecedentes, las cuales por su rigor científico constituyen un referente con aportes valiosos para profundizar el análisis del fenómeno aquí planteado y sus variables.

\section{Participantes}

La muestra poblacional está conformada por 49 casos de intentos de suicidios presentados en adolescentes de 12 a 17 años de edad en la ciudad de Sincelejo, victimas y no víctimas del conflicto armado, registrados en la Secretaria de Salud Departamental de Sucre durante el año 2017.

\section{Instrumentos}

Mediante una matriz de registro, se seleccionaron y organizaron los casos, tomando en cuenta las variables sociodemográficas.

\section{Análisis de la información}

El análisis de los datos se realizó de manera descriptiva empleando el programa SPSS, el cual es un software utilizado para la captura y análisis de datos.

\section{Resultados}

Los resultados de la investigación se presentan mediante tablas de frecuencias y figuras, en atención a las variables sociodemográficas. Seguido del análisis descriptivo de cada una de ellas, destacando aquellos con mayor y menor porcentaje. Finalmente, se procedió a identificar las características psicosociales de los adolescentes víctimas y no víctimas de desplazamiento.

\section{Variables Sociodemográficas}

El grupo de adolescentes registrados con intentos de suicidio durante el año 2017 se conformó por 43 mujeres correspondiente al 87,8\% y 6 hombres equivalentes al $12,24 \%$ de la muestra poblacional, del 87,8\% 18 
adolescentes fueron víctimas y 25 no víctimas del conflicto armado y donde el sexo femenino resultó con mayor intentos de suicidio para ambos grupos.

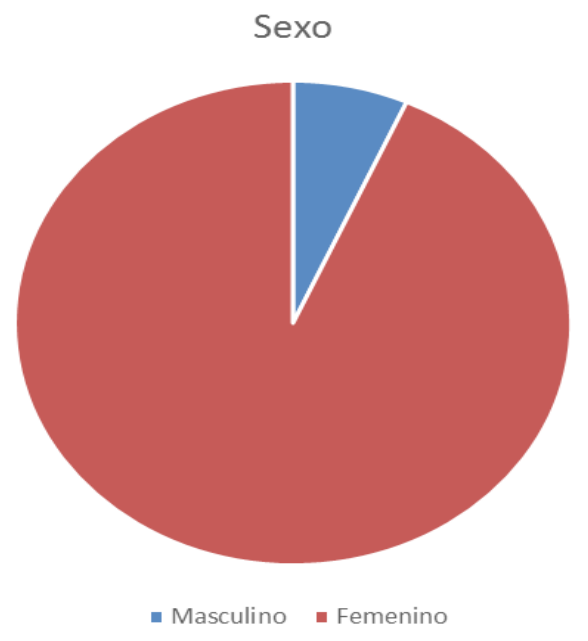

Figura 1. Sexo de la muestra.

Según la distribución de adolescentes por edad, se resalta el grupo situado entre 12 y 15 años que presentaron un 20,4\% y 24,5\% de los casos, seguido del grupo de 14 y 17 años con un 16,3\% y 18,4\%, lo que expresa que los intentos prevalecieron en el rango de menor edad.

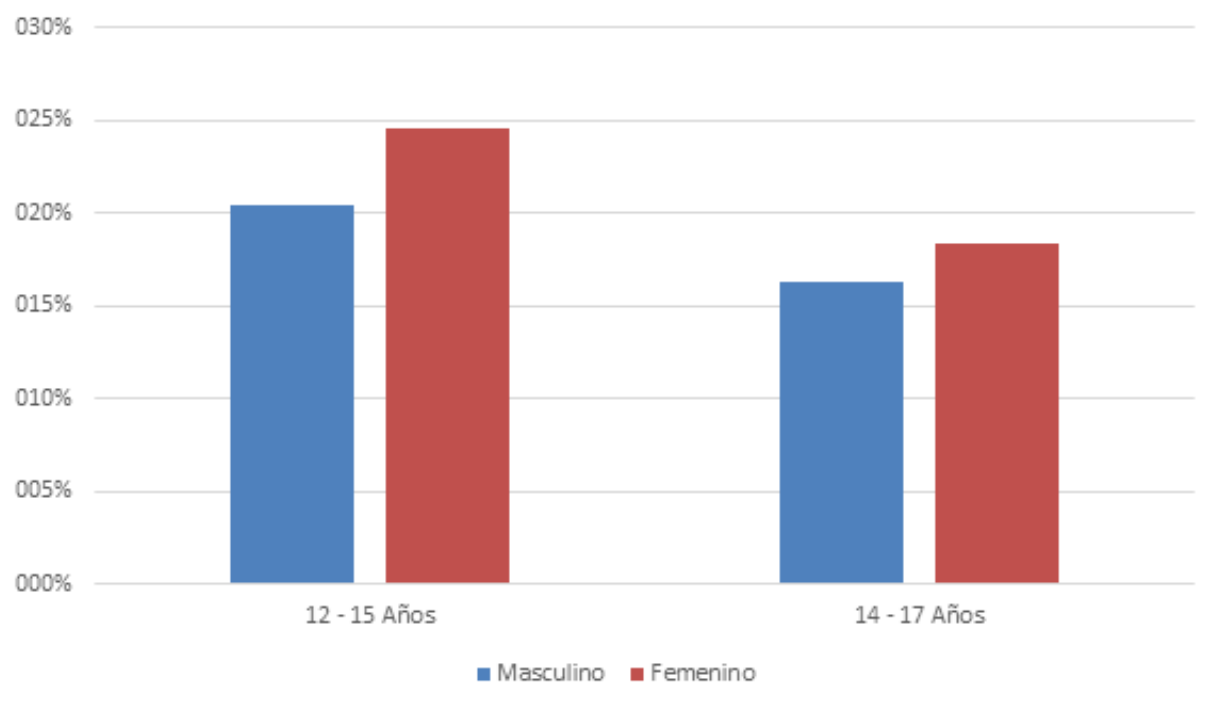

Figura 2. Edades de la muestra. 
Los grupos estudiados no registran problemas legales, en cambio sí presentaron problemas de pareja $(10,2 \%)$ y en menor porcentaje problemas económicos, muerte de familiar y enfermedades crónicas registrando 2,0\%. Esta información se encuentra de manera más detallada en la Tabla 1.

\section{Tabla 1}

Características Sociodemográficas

\begin{tabular}{|c|c|c|c|c|c|c|}
\hline & \multicolumn{2}{|c|}{ Total } & \multicolumn{2}{|c|}{ Víctimas } & \multicolumn{2}{|c|}{ No Víctimas } \\
\hline & $\mathrm{N}$ & $\%$ & $\mathrm{~N}$ & $\%$ & $\mathrm{~N}$ & $\%$ \\
\hline \multicolumn{7}{|c|}{ Sexo } \\
\hline Chicas & 43 & 87,8 & 18 & 90 & 25 & 86,2 \\
\hline Chicos & 6 & 12,2 & 2 & 10 & 4 & 13,8 \\
\hline \multicolumn{7}{|c|}{ Edad } \\
\hline 12 & 10 & 20,4 & 5 & 25 & 5 & 17,2 \\
\hline 13 & & 8,2 & 2 & 10 & 2 & 6,9 \\
\hline 14 & 8 & 16,3 & 4 & 20 & 4 & 13,8 \\
\hline 15 & 12 & 24,5 & 3 & 15 & 9 & 31,0 \\
\hline 16 & 6 & 12,2 & 3 & 15 & 3 & 10,3 \\
\hline 17 & 9 & 18,4 & 3 & 15 & 6 & 20,7 \\
\hline \multicolumn{7}{|c|}{ Problema pareja } \\
\hline Sí & 5 & 10,2 & 2 & 10,0 & 3 & 10,3 \\
\hline No & 44 & 89,8 & 18 & 90,0 & 26 & 89,7 \\
\hline \multicolumn{7}{|c|}{ Enfermedad crónica } \\
\hline Sí & 1 & $2,0 \%$ & - & - & 1 & 3,4 \\
\hline No & 48 & $98,0 \%$ & 20 & 100 & 28 & 96,6 \\
\hline \multicolumn{7}{|c|}{ Problemas económicos } \\
\hline Sí & 1 & 2,0 & 1 & 5,0 & 7 & 24,1 \\
\hline No & 38 & 77,6 & 16 & 8,0 & 22 & 75,9 \\
\hline \multicolumn{7}{|c|}{ Muerte familiar } \\
\hline Sí & 1 & 2,0 & 3 & 15 & 1 & 3,4 \\
\hline No & 38 & 77,6 & 17 & 85 & 21 & 72,4 \\
\hline \multicolumn{7}{|c|}{ Problemas legales } \\
\hline Sí & - & - & - & - & - & - \\
\hline No & 49 & 100 & 20 & 100 & 29 & 100 \\
\hline
\end{tabular}

Fuente: elaboración propia. 


\section{Características de los intentos de suicidio de los participantes}

En cuanto a las características del intento suicida sobresalen 17 intentos previos equivalente a $34,7 \%$ de los adolescentes estudiados, de los cuales $8(16,32 \%)$ son víctimas y 9 no víctimas (18,36\%). Lo que indica que los adolescentes que atentan contra su vida tienen un significativo número de intentos previos y el mayor porcentaje corresponde adolescentes no víctimas del conflicto armado.

Así mismo, se presentaron dos antecedentes de suicidio en la familia, uno por cada grupo sumando 4,1\%. Otra de las características reflejadas en los adolescentes fue la presencia de ideación suicida, reportando 11 casos que equivalen al 22,4\% distribuido en 6 adolescentes víctimas y 5 no víctimas.

Con relación a los tipos de intento que más utilizaron los adolescentes contra su vida, se encuentra en mayor porcentaje los casos por intoxicación $(61,25 \%)$ donde 13 fueron víctimas $(65,0 \%)$ y 17 no victimas $(58,6 \%)$, seguido por arma corto punzante, hallándose 15 casos con $30,6 \%$, de los cuales 6 adolescentes víctimas y 9 no víctimas. En menor frecuencia se registraron casos por lanzamiento al vacío y ahorcamiento. Es necesario precisar que ninguno de los casos estudiados intentó suicidarse con lanzamiento a vehículos, arma de fuego e inmolación. Esto se amplía en la Tabla 2.

Tabla 2

Características del Intento Suicida

\begin{tabular}{lcccccc}
\hline & \multicolumn{2}{c}{ Total } & \multicolumn{3}{c}{ Víctimas } & \multicolumn{2}{c}{ No Víctimas } \\
& $\mathbf{N}$ & $\%$ & $\mathbf{N}$ & $\%$ & $\mathbf{N}$ & $\%$ \\
\hline Sí & 17 & 34,7 & 8 & 40 & 9 & 31,0 \\
NO & 32 & 65,3 & 12 & 60 & 20 & 69,0 \\
\hline \multicolumn{6}{c}{ Número de intentos } \\
1 & 5 & 10,2 & 2 & 10,0 & 3 & 10,3 \\
2 & 3 & 6,1 & 1 & 5,0 & 2 & 6,9 \\
3 & 4 & 8,2 & 1 & 5,0 & 3 & 10,3 \\
4 & 4 & 8,2 & 3 & 15,0 & 1 & 3,4 \\
\hline
\end{tabular}




\begin{tabular}{|c|c|c|c|c|c|c|}
\hline & \multicolumn{2}{|c|}{ Total } & \multicolumn{2}{|c|}{ Víctimas } & \multicolumn{2}{|c|}{ No Víctimas } \\
\hline & $\mathbf{N}$ & $\%$ & $\mathbf{N}$ & $\%$ & $\mathrm{~N}$ & $\%$ \\
\hline \multicolumn{7}{|c|}{ Suicidio Familia } \\
\hline SÍ & 2 & 4,1 & 1 & 5,0 & 1 & 3,4 \\
\hline NO & 37 & 75,5 & 16 & 80,5 & 21 & 72,2 \\
\hline Perdidos & 10 & 20,4 & 3 & 15,0 & 7 & 24,1 \\
\hline \multicolumn{7}{|c|}{ Ideación suicida } \\
\hline Sí & 11 & 22,4 & 6 & 30,0 & 5 & 17,2 \\
\hline NO & 38 & 77,6 & 14 & 70,0 & 24 & 82,8 \\
\hline \multicolumn{7}{|c|}{ Ahorcamiento } \\
\hline Sí & 2 & 4,1 & 1 & 5,0 & 1 & 3,4 \\
\hline NO & 47 & 95,9 & 19 & 95 & 28 & 96,6 \\
\hline \multicolumn{7}{|c|}{ Arma Corto punzante } \\
\hline SÍ & 15 & 30,6 & 6 & 30 & 9 & 31,0 \\
\hline $\mathrm{NO}$ & 34 & 69,4 & 14 & 70 & 20 & 69 \\
\hline \multicolumn{7}{|c|}{ Arma de fuego } \\
\hline SÍ & - & - & - & - & - & - \\
\hline NO & 49 & 100 & 20 & 100 & 29 & 100 \\
\hline \multicolumn{7}{|c|}{ Inmolación } \\
\hline Sí & & & - & - & - & - \\
\hline NO & 39 & 79,6 & 17 & 85,0 & 22 & 75,9 \\
\hline \multicolumn{7}{|c|}{ Lanzamiento al vacío } \\
\hline SÍ & 1 & 2,0 & - & - & 1 & 3,4 \\
\hline NO & 48 & 98,0 & 20 & 100 & 29 & 100 \\
\hline \multicolumn{7}{|c|}{ Lanzamiento vehículo } \\
\hline Sí & - & - & - & - & - & - \\
\hline NO & 49 & 100 & 20 & 100 & 29 & 100 \\
\hline \multicolumn{7}{|c|}{ Intoxicación } \\
\hline Sí & 30 & 61,2 & 13 & 65,0 & 17 & 58,6 \\
\hline NO & 19 & 38,8 & 7 & 35,0 & 12 & 41,4 \\
\hline
\end{tabular}

Fuente: elaboración propia 


\section{Aspectos psicológicos de los adolescentes de la muestra}

Respecto a las características psicológicas de los participantes, predominó la depresión como el trastorno psicológico que registraron con mayor frecuencia los adolescentes (con 22,4\%), de los cuales 5 (25\%) fueron víctimas y $6(20,7 \%)$ no víctimas del conflicto armando. Por su lado, la esquizofrenia registró un caso en adolescentes victimas igual a $5,0 \%$. Cabe anotar, que no se presentaron trastornos de personalidad y bipolaridad, tal como se registra en la Tabla 3.

Tabla 3

Características Psicológicas de los participantes

\begin{tabular}{|c|c|c|c|c|c|c|}
\hline \multirow[b]{2}{*}{$\begin{array}{c}\text { Trastornos } \\
\text { psicológicos }\end{array}$} & \multicolumn{2}{|c|}{ Total } & \multicolumn{2}{|c|}{ Víctimas } & \multicolumn{2}{|c|}{ No Víctimas } \\
\hline & $\mathrm{N}$ & $\%$ & $\mathbf{n}$ & $\%$ & $\mathrm{~N}$ & $\%$ \\
\hline \multicolumn{7}{|c|}{ Trastorno depresivo } \\
\hline Sí & 11 & 22,4 & 5 & 25 & 6 & 20,7 \\
\hline NO & 5 & 10,2 & 1 & 5,0 & 4 & 13,8 \\
\hline Perdidos & 33 & 67,3 & & & & \\
\hline \multicolumn{7}{|c|}{ Trastornos de personalidad } \\
\hline Sí & & & - & - & - & - \\
\hline NO & 6 & 12,2 & 3 & 15,0 & 3 & 10,3 \\
\hline Perdidos & 43 & 87,8 & 17 & 85 & 26 & 89,7 \\
\hline \multicolumn{7}{|c|}{ Trastorno bipolar } \\
\hline SÍ & & & - & - & - & - \\
\hline NO & 16 & 32,7 & 6 & 30 & 10 & 34,5 \\
\hline Perdidos & 33 & 67,3 & 14 & 70 & 19 & 65,5 \\
\hline \multicolumn{7}{|c|}{ Esquizofrenia } \\
\hline SÍ & 1 & 2,0 & 1 & 5,0 & - & - \\
\hline $\mathrm{NO}$ & 15 & 30,6 & 5 & 25 & 10 & 34,5 \\
\hline Perdidos & 33 & 67,3 & 14 & 70,0 & 19 & 65,5 \\
\hline
\end{tabular}

Fuente: elaboración propia. 


\section{Discusión y conclusiones}

La adolescencia es una etapa en la que ocurren la mayor parte de las transformaciones y cambios en cuanto al desarrollo psicológico, neuroendocrinológico y social (Gilchrist, 1991). Es así como dentro de esta etapa existen factores de riesgo y problemáticas que pueden afectar a los adolescentes a nivel emocional y psicosocial, donde la edad, el género y la depresión son factores asociados al intento de suicidio.

En función del análisis de los resultados en la presente investigación, y teniendo en cuenta los objetivos, se debe mencionar que la muestra poblacional femenina es predominante a comparación de la masculina; lo cual indica que para esta población, el género femenino presentó mayor índice de intentos de suicidio respecto al masculino. Castro (2001) considera que existe en cierta medida mayor índice de suicidios en mujeres y que este se encuentra asociado a dificultades amorosas y familiares como principales causas, al igual que a la aparición en segundo plano de trastorno mental.

En este mismo sentido, se considera que en los países occidentales, las adolescentes y las mujeres jóvenes son más propensas que los hombres a divulgar el comportamiento suicida, sin embargo, los hombres son más propensos a suicidarse respecto a las mujeres. A esto se le suma que el consumo de sustancias psicoactivas en la adolescencia o incluso la ansiedad por separación o las rupturas de pareja puede incrementar en mayor medida el riesgo de que ambos sexos intenten el suicidio (Cabra, Infante, Sossa, 2010). En efecto, en Sucre se halló una relación significativa entre el consumo de sustancias y la depresión; la sustancia más consumida por los jóvenes fue el alcohol (Romero-Acosta, Gómez-de-Regil, Lowe \& Lipps 2018). Esto es importante debido a que hay una relación estrecha entre la depresión y la conducta suicida.

Así mismo, la edad promedio de los participantes es de 14.66 años. En este grupo poblacional los intentos de suicidio prevalecieron en el rango de 12 a 15 años de edad, situación que se encuentra en coherencia con lo expuesto por Cabra, et al. (2010), quienes mencionan que en Colombia los índices de intento de suicidio son mayoritarios para sexo femenino y dentro del rango de edad de 10 a 14 años, a diferencia de los reportes publicados en los Estados Unidos, donde la franja se mantiene superior para la población masculina. 
El Servicio de Psiquiatría Pediátrica en Argentina señala que las conductas e intentos suicidas son más frecuentes en adolescentes que se encuentran en edades tempranas y no se debe exactamente a la presencia de trastornos psicopatológicos, ya que se encuentran más relacionados con problemáticas familiares, de pareja o por trastorno depresivo, bipolar y de conducta antisocial, afirmación que nutre el supuesto de esta investigación, pues en cuanto a las características asociadas al suicidio, las problemáticas de pareja se hicieron evidentes en mayor medida en los adolescentes participantes de esta investigación, a comparación de los problemas económicos, familiares o por enfermedades crónicas. De modo similar, para Gómez et al. (2002) la población que se encuentra en mayor riesgo está entre los 16 a 21 años de edad; presenta relación con una combinación de eventos adversos de la vida, acontecimientos vitales estresantes y trastorno mental.

De los datos obtenidos correspondientes a las características del intento suicida, se encontró que en un porcentaje significativo de la población existieron intentos previos de suicidio e ideación suicida y suicidio de familiares, hallazgo semejante a lo propuesto por Shaffer (2001) quien señaló que haber cometido intentos previos incrementa mucho más el riesgo de suicidio, pero este efecto predictivo es menor para el sexo femenino. De modo similar, este fenómeno se encuentra influenciado por diversas variables biológicas, genéticas, psicológicas, culturales, económicas y sociales, las cuales se pueden manifestar en diferentes áreas y niveles como el micro individual, el meso familiar, el escolar y macrosocial. Entre ellos se pueden destacar a nivel individual: los intentos previos de suicidio; conflictos de pareja o finalización de una relación amorosa; desesperanza; enfermedad crónica y/o discapacidad; predisposición genética; trastorno mental; conductas violentas y autodestructivas; conflictos de identidad; consumo de alcohol y de sustancias psicoactivas.

Dentro del componente Meso familiar existen factores como: la violencia física, sexual, psicológica, la negligencia, el abuso de alcohol o de drogas por parte de miembros de la familia, antecedentes familiares de suicidio o de intentos suicidas, trastorno mental, dificultades socioeconómicas, muerte de algún familiar cercano y la separación o el divorcio. 
A nivel exo macro, también existen factores de riesgos asociados a los intentos suicidas como: la carecían de redes de apoyo, acceso a armas de fuego, discriminación, dificultades para acceder a servicios, violencia sexual y ser víctima del conflicto armado. Elementos que coinciden con lo encontrado en la presente investigación, donde un porcentaje significativo de la población adolescente es víctima del conflicto armado, ya que en cualquiera de sus hechos victimizantes y especialmente dentro de la violencia sexual, puede traducirse en complicaciones serias, que causan mucha afectación en la vida y en el desarrollo de las víctimas y puede tener una estrecha relación con el suicidio.

En esta misma línea, también se encontró coincidencias con lo planteado por Bedoya y Montaño (2015), quienes indicaron en sus hallazgos que la presencia de un trastorno mental está asociado como un factor de riesgo para cometer un suicidio. Entre los trastornos que más se asocian como riesgo del suicidio se encuentran la depresión y la esquizofrenia, situación característica para esta población, en este caso predominó la presencia en mayor medida de trastorno depresivo.

Adicionalmente, resulta importante destacar que la disfunción familiar es un factor de riesgo determinante, ya que distintas investigaciones mencionan que en la mayor parte de las familias de adolescentes que realizaron intento suicida, existen problemáticas familiares y crisis en el hogar, predominando la desorganización, desmoralización, al igual que el deterioro de las relaciones familiares (Nizama, 2011), por lo cual se recomienda ampliar esta línea temática de investigación.

Por otro lado, los métodos de intento que más utilizaron los adolescentes contra su vida fue la intoxicación, y las armas corto punzantes, prevaleciendo en los participantes no víctimas del conflicto armado. De modo similar el ICBF, (2018) señaló que los métodos más empleados por la población menor de 18 años para llevar a cabo el suicidio son intoxicación y arma de fuego, lo que se encuentra en coherencia con los resultados obtenidos. Alvis, Soto y Grisales (2017), encontraron que el mecanismo de intento de suicidio más común y utilizado por los jóvenes son las intoxicaciones, seguido por las armas cortopunzantes. Diversos estudios han señalado hallazgos similares. Sabadell y Terrassa, citado por Cebria et al. (2012), mencionaron que las personas en su mayoría han usado la sobredosis de medicamentos como método para realizar el intento de 
suicidio, en similitud a esto, también Lin, et al. (2014) identificaron que el método más usado ha sido el envenenamiento o la intoxicación.

En tal sentido, el intento de suicidio por intoxicaciones voluntarias representa una problemática de mucha relevancia entre los adolescentes, sobre todo en el sexo femenino (Garrido, et al. 2000), debido a que se ha incrementado sustancialmente en los últimos años y es una situación con altas incidencias en la adolescencia temprana y para las féminas.

A pesar de que la problemática fue más prevalente en los adolescentes no víctimas del conflicto armado, en los resultados obtenidos no se evidenciaron diferencias significativas para ambos grupos, esto podría deberse a que el mayor número de participantes corresponde a adolescentes no víctimas.

En definitiva, el intento suicida es un fenómeno multifactorial que puede estar relacionado con agentes biológicos, psicológicos y problemáticas propias de la sociedad actual, por lo cual, es de gran importancia promover el empoderamiento de las comunidades frente al tema, para mayor claridad de los factores de riesgo y sus formas de intervención. Por ello, es necesario considerarlo como un problema de salud pública y abordarlo como bien lo proponen Bronfenbrenner, desde una perspectiva holística e integradora, que contemple el análisis de los diversos contextos en los que se desarrollan los adolescentes y la forma en la que interactúan con los mismos. Identificando cada una de las necesidades que presenten las personas, familias o comunidades, evitando así la presencia de factores de riesgo que posiblemente conlleven a desencadenar conductas suicidas.

La anterior investigación permite llegar a la conclusión que la etapa de la adolescencia es el grupo etario que se encuentra en mayor riesgo de cometer intentos de suicidio, lo que representa un dato relevante que se debe tener en cuenta en posteriores investigaciones, para así, hallar resultados que evidencien ante las entidades gubernamentales, un referente para trazar lineamientos tendientes al fortalecimiento de políticas públicas a favor de la calidad en los programas de salud mental, con acciones preventivas y de articulación intersectorial oportuna y eficaz, pero sobre todo una atención humanizada que atiendan las necesidades psicológicas de los adolescentes, disminuyendo en gran parte los factores de riesgo de intentos de suicidio y suicidios en este grupo etario. 


\section{Referencias}

Alvis, L. F., Soto, \& Grisale, H. (2017). El intento de suicidio en Ibagué: el silencio de una voz de auxilio. Revista Criminalidad, 59(2), 81-92.

Bedoya, E., \& Montaño, L. (2016). Suicidio y Trastorno Mental. Rev. CES Psicol., 9(2), 179-201. Recuperado de: http://www.redalyc.org/ pdf/4235/423548400012.pdf.

Cabra, O., Infante, D. y Sossa, F. (2010). El suicidio y los factores de riesgo asociados en niños y adolescentes. Revista Médica Sanitas: 13(2): 28-35.

Castro, J. (2001). Intentos de suicidio atendidos en el Hospital Psiquiátrico "Villahermosa. Salud en Tabasco, 7 (1), 363-365. Recuperado de: http://www.redalyc.org/pdf/487/48770106.pdf.

Cebrià, A. I., Parra, I., Pàmias, M., Escayola, A., García-Parés, G., Puntí, J., ... \& Hegerl, U. (2013). Effectiveness of a telephone management programme for patients discharged from an emergency department after a suicide attempt: controlled study in a Spanish population. Journal of affective disorders, 147(1-3), 269-276.

Corona, M., Hernández, M., García, R. (2016). Mortalidad por suicidio, factores de riesgos y protectores. Revista habanera de Ciencias Médicas, 15 (1). 90-100.

El Mundo.com. (2017). Depresión: la enfermedad mental más frecuente en Colombia. Recuperado de https://www.elmundo.com/noticia/Depresionla-enfermedad-mental-mas-frecuente-en-Colombia/361777.

Garrido, R., García, J., Carballo, E., Luaces, C., Alda, J., y Pou, J. (2000). Intoxicaciones voluntarias como intento de suicidio: Voluntary intoxication As A Form Of Attempted Suicide. Anales de Pediatría: Vol. 53. Pag. 213-216.

Gilchrist V.J. (1991). Preventive health care for the adolescent; En: Am. Fam. Physician, 43(3), pp.869-78, Canada, March, 1991.

Gómez, C., Rodríguez, N., Bohórquez, A., Diazgranados, Nancy., Ospina, M., Fernández, C. (2002). Factores asociados al intento de suicidio en la Población Colombiana. Revista Colombiana de Psiquiatría. 31, (4), 271- 286. 
Hernandez, R., Fernandez, C., \& Baptista, P. (2010). Metodologia de la investigación. Mexico: McGraw Hill.

Lin, C. J., Lu, H. C., Sun, F. J., Fang, C. K., Wu, S. I. \& Liu, S. I. (2014). The characteristics, management, and aftercare of patients with suicide attempts who attended the emergency department of a general hospital in northern Taiwan. Journal of the Chinese Medical Association, 77 (6): 317-324.

Nizama, M. (2011). Suicidio. Revista Peruana de Epidemiología, 15 (2), 81-85. Recuperado de: http://www.redalyc.org/comocitar. oa?id=203122516002.

Observatorio del Bienestar de la Niñez - OBN. (2017). Insumos para la prevención de la violencia sexual en Colombia. En línea, disponible en: https://www.icbf.gov.co/sites/default/files/_boletin_1-violencia_ sexual.pdf.

Restrepo, H.J., Romero-Acosta, K., Verhelst-Montenegro, S. (2019). Caracterización del Suicidio en el departamento de Sucre: Un análisis de las noticias publicadas en el Periódico El Meridiano de Sucre, Colombia. Búsqueda, 6(22):e426. DOI: https://doi. org/10.21892/01239813.426.

Romero-Acosta, K., Gómez-de-Regil, L., Lowe, G., \& Lipps, G. (2018).The Relationship between Substance Use and Depressive Symptoms among Colombian Adolescents, West Indies Medical Journal, 67, (4), 323-7. DOI 10.7727/wimj.2018.143.

Shaffer, D. (2001). Practice parameter for the assessment and treatment of children and adolescents with suicidal behavior, julio 2001. 